International Journal of Distributed and Parallel Systems (IJDPS) Vol.3, No.4, July 2012

\title{
A UNIFIED COMPARISON OF CDMA EVDO KEY Performance Parameters in Multi-Vendor ENVIRONMENT
}

\author{
Rabnawaz Anwar ${ }^{1}$ and Irfan Ahmed ${ }^{2}$ \\ ${ }^{1}$ Institute of Communications Technologies (ICT-PTCL) \\ NWFP University of Engineering and Technology Peshawar, Pakistan \\ rabnawaz . anwarehotmail. com \\ ${ }^{2}$ College of Computers and Information Technology \\ Taif University, P.O. Box 888 Taif, Saudi Arabia \\ i.ahmedetu.edu.sa
}

\begin{abstract}
With the standardization of CDMA2000-1x, EVDO, and its subsequent release/revisions $(0, A, B$, and $C)$ each telecom vendor continues to develop their equipment as well as network management software for supporting EVDO services and defines EVDO performance parameters separately. This leads to sort of redundancy and perplexity in multivendor network environments in which network optimization engineer has to deal with all these performance parameters to optimize the system performance. There is need to unify these different EVDO performance parameters defined by different vendors to overcome this redundancy and confusion. To complete this objective we first has identified different EVDO key performance indicators defined by different vendors through their software i.e. element management systems (EMS). After identification of these KPIs we then take one most important KPI i.e., the Session Setup Success Rate (SSSR) and analyses its constituent counters and find out differences through daily statistic reports, standard definitions formula and message flow diagrams defined by each vendor. Then we proposed a new KPI, HSN Ratio (Harmonic mean of session setup success and session negotiation success Ratios) to get a complete picture of end-to-end session setup success rate. Finally we have collected 15 days hourly statistic report of SSSR and session negotiation success rate defined by each vendor through their EMS's and applied minimum mean square error method to unify the proposed HSN ratio. As a result of this process we have figure out differences between multivendor KPIs and minimizes these differences to unify them. Similarly this whole process could be repeated to unify other EVDO KPIs.
\end{abstract}

\section{KEYWORDS}

CDMA, EVDO, KPI; SSSR

\section{INTRODUCTION}

CDMA2000 1xEVDO is a third generation wireless data technology standard accepted by third Generation Partnership Project 2 (3GPP2) as a solution to provide data services in the wide area mobile and fixed networks [1]. It is based on High data rate concept introduce in [2]. Initially it is released as CDMA2000 1xEVDO Revision 0 having peak rates of $2.45 \mathrm{Mbps}$ in downlink and $153.6 \mathrm{Kbps}$ in uplink. But with the increasing demand of high speed wireless internet access resulted in rapid growth of number of EVDO users. Operators have observed strong demand for applications like VOIP, video telephony, wireless gaming .rich multimedia services along with downlink intensive applications such as web browsing and file transfer. These applications demand system that can support large number of simultaneous users and their latency requirements. In order to meet this demand, 3GPP2 approved enhancements to CDMA 2000 revision 0 (TIA-856). CDMA2000 1xEVDO Revision A (TIA-856-A) was therefore 
International Journal of Distributed and Parallel Systems (IJDPS) Vol.3, No.4, July 2012

standardized in March 2004 by 3GPP2 and TIA. It meets downlink intensive applications by introducing several new concepts as H-ARQ in reverse link, higher modulation, reverse resource control, Seamless virtual soft handoff and rapid connection as depicted in [3]. With the advent of these new concepts and technologies the peak rates increases up to $3.1 \mathrm{Mbps}$ in downlink and 1.8Mbps in reverse link for CDMA 2000 Revision A.

As with the standardization of EVDO Rev A each telecom vendor continues to develop their equipment as well as software for supporting EVDO Rev A services and defines EVDO Revision A performance parameters separately. Performance parameters are set of entities that provide real time system performance data to measure monitor and evaluate performance in the network. With the help of these performance parameters we can engineer the system resources, optimize the system performance and improve the quality of service. Performance parameters are very important in dealing with any telecom network. With the help of these parameters a network is optimize for offering up to mark services. It enables to collect real time performance data and then this data is used to evaluate and analyze system and network performance. Most of the network service providers around the world incorporate multivendor equipment in their networks to limit the vendor monopoly. So telecom engineers of the service provider have to deal with various performance parameters defined separately by each vendor although the technology i.e. EVDO is same. This creates confusion for telecom engineers/end users of this software by referring to each vendor specifications when optimizing the network. So there is a need of identification \& unification of these vendor specific performance parameters to one that are defined by 3GPP2.In this way Network Engineers have to deal with one set of performance parameters even in multivendor environment. This also helps in clarifying as well as quickly identifying different problems related to their networks and helps in quickly proposing solution for the problem.

Identification and unification of EVDO Rev A performance parameters is a practical problem faced by telecom service providers that have multivendor telecom equipment installed in their networks. Due to its practical nature this area is not explored before. Most of the research work done before related to performance parameter of EVDO is done by each vendor by defining parameters exclusively for their own equipment's. Frances Jiang Feb 2004 of Lucent Technologies analyses EVDO service measurements for call scenarios and capacity measurement [4]. In his work he identified standard Key Performance Indicators of EVDO and performance metrics related to it.

Our ultimate goal is to identify different EVDO Revision A performance parameters defined by different vendors (as in [8] \& [9]) using their software for network optimization and analysis. These different parameters are then compared and analyzed by different mathematical calculations and simulations in MATLAB $^{\circledR}$ and then finally a set of unified performance parameters are formulated. At last conclusions and future work in the area is presented.

\section{EVDO CALl SETUP}

When an access terminal (AT) originates a data call, it has to pass through BTS, PCF, AAA, and PDSN before going to Internet cloud as shown in Figure 1. A brief function of each of the network element is given below. 


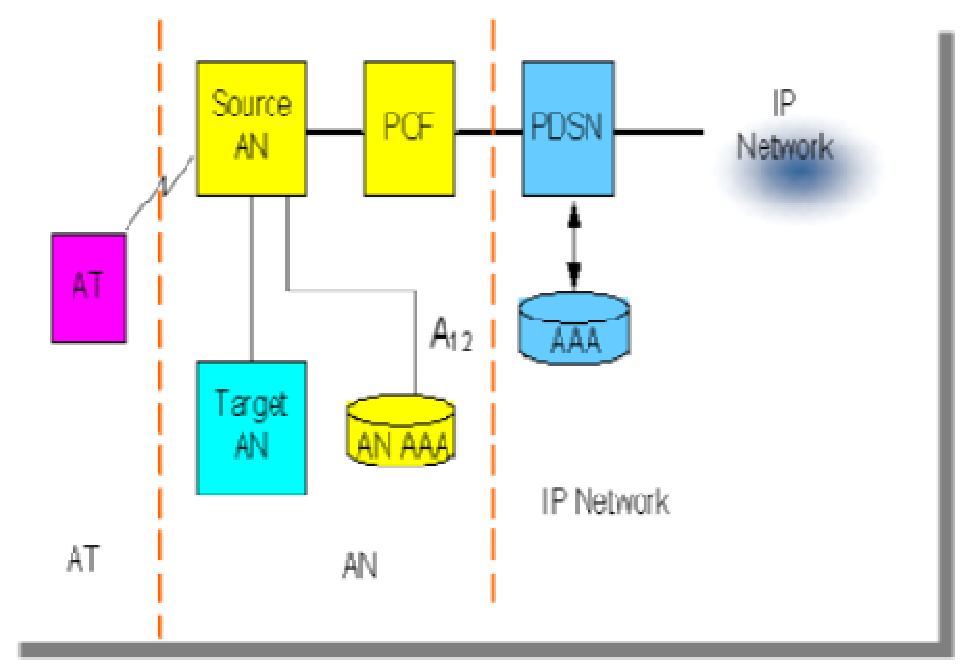

Figure 1. EVDO Network Architecture

Access Terminal (AT) provides data connectivity to a user. An access terminal may be connected to a computing device such as a laptop personal computer or it may be a selfcontained data device such as a personal digital assistant. An access terminal is equivalent to a mobile station.

Access Network (AN) is equipment providing data connectivity between a packet switched data network (typically the Internet) and the access terminals. An access network is equivalent to a base station.

Packet Control Function (PCF) is an entity in the radio access network that manages the relay of packets between the AN and the PDSN.

Packet Data Serving Node (PDSN) acts as an interface between Radio Network and Packet Data Network. It provides cdma2000 mobile station with services for Internet access or Intranet access. It provides the mobile station with Simple IP access service or Mobile IP access service. In Simple IP, PDSN acts as a Network access server (NAS) In Mobile IP, PDSN acts as Foreign Agent (FA) for Mobile Station. It acts as a client of AAA server.

Authentication Authorization and Accounting (AAA) server is a server program that handles user requests for access to computer resources and, for an enterprise, provides authentication, authorization, and accounting (AAA) services. The AAA server typically interacts with network access and gateway servers and with databases and directories containing user information. The current standard by which devices or applications communicate with an AAA server is the Remote Authentication Dial-In User Service (RADIUS). Types of AAA servers used in EVDO are Access Network AAA (AN-AAA), Home AAA (H-AAA) and Visited AAA (V-AAA).

\subsection{AT Originated Call Setup Steps}

This section describes the call flow for a successfully authenticated AT call origination in the AN as depicted in Figure 2.The detail of each step is given below [2]. 
International Journal of Distributed and Parallel Systems (IJDPS) Vol.3, No.4, July 2012

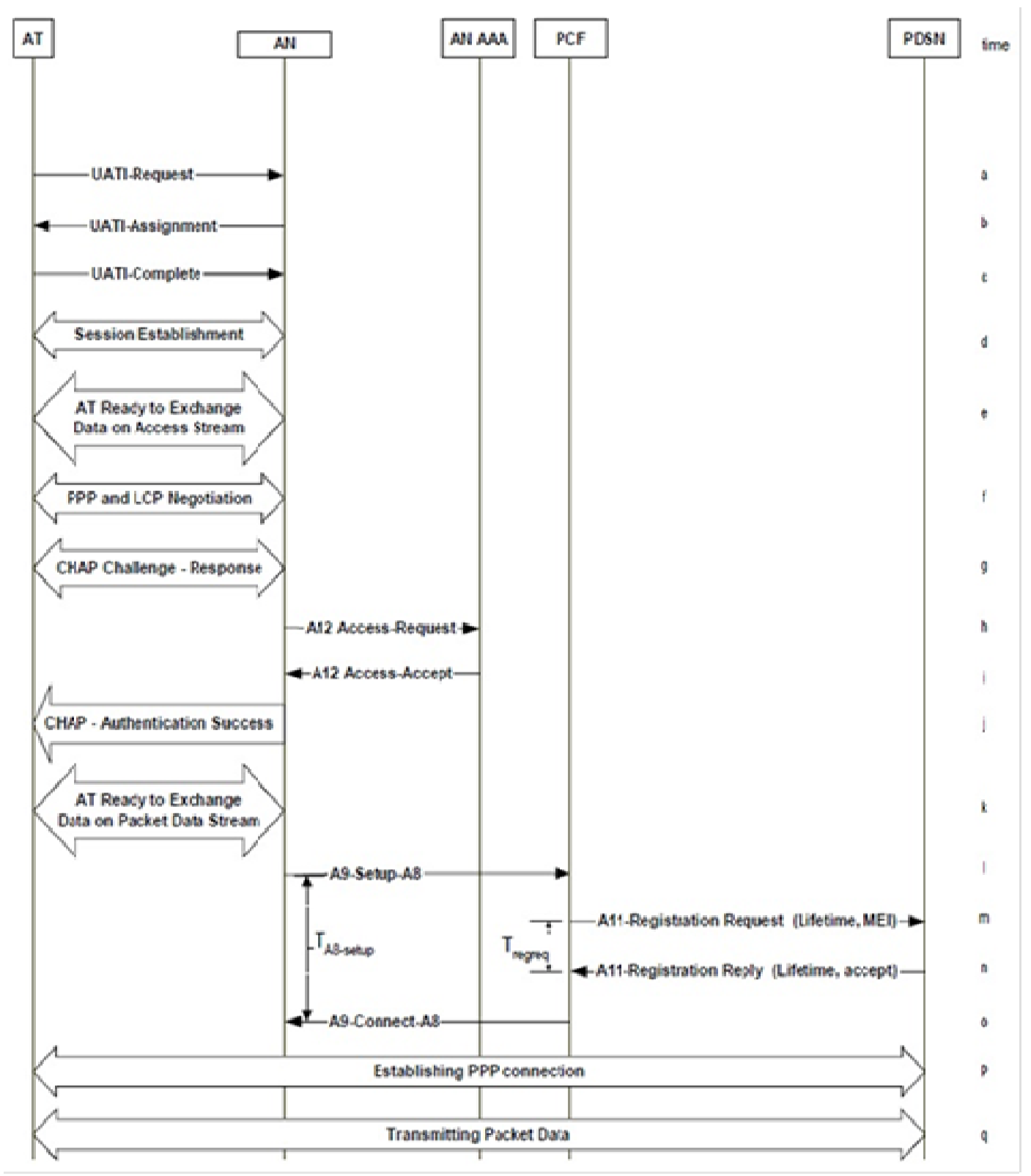

Figure 2. 1xEVDO Call Setup-Successful Authentication

a. The AT sends a UATI-Request message to request that a Unicast Access Terminal Identifier (UATI) be assigned to it by the AN.

b. The AN sends a UATI-Assignment message to assign a UATI to the AT.

c. The AT sends a UATI-Complete message to notify the AN that it has received the UATI-Assignment message. 
International Journal of Distributed and Parallel Systems (IJDPS) Vol.3, No.4, July 2012

d. If no session exists between the AT and AN, a session is established where protocols and protocol configurations are negotiated, stored and used for communications between the AT and the AN. Refer to TIA/EIA/IS-856, Section 5, Session Layer.

e. The AT indicates that it is ready to exchange data on the access stream (e.g., the flow control protocol for the default packet application bound to the AN is in the open state).

f. The AT and the AN initiate Point-to-Point Protocol (PPP) and Link Control Protocol (LCP) negotiat 12 ions for access authentication. Refer to RFC 1661.

g. The AN generates a random challenge and sends it to the AT in a Challenge Handshake Authenticat14 ion Protocol (CHAP) Challenge packet in accordance with RFC 1994.

h. When the AN receives the CHAP response packet from the AT, it sends a RADIUS Access-Request message packet on the A12 interface to the AN Authentication, Authorization and Accounting (AAA) entity (which acts as a RADIUS server) in accordance with RFC-2865.

i. The AN-AAA looks up a password based on the User-name attribute in the A12 Access-Request and if the authentication passes (as specified in RFC-1994 and RFC2865), the AN AAA sends an Access-Accept packet on the A12 interface. The A12 Access-Accept contains a RADIUS attribute with Type set to 20 (Callback-Id). Refer to Section 4.3.2, RADIUS Support.

j. The AN returns an indication of CHAP authentication success, to the AT. Refer to RFC 1994.

k. The AT indicates that it is ready to exchange data on the packet data stream. (E.g., the flow control protocol for the default packet application bound to the packet data network is in the open state).

1. The AN sends an A9-Setup-A8 message to the Packet Control Function (PCF) and starts timer TA8- setup, to establish the A8-Connection. The A9-Setup-A8 message shall not be sent before the AT indicates that it is ready to exchange data on the access stream, as identified in step e.

m. The PCF recognizes that no A10 connection associated with the AT is available and selects a PDSN. The PCF sends an A11-Registration Request message to the PDSN which includes the Mobility Event Indicator (MEI) within the Vendor/Organization Specific Extension. The PCF starts timer Tregreq.

n. The A11-Registration Request is validated and the PDSN accepts the connection by returning an A11-Registration Reply with an accept indication and Lifetime set to the configured Trp. Both the PDSN and the PCF create a binding record for the A10 connection. The PCF stops timer Tregreq.

o. When the AN receives the A9-Connect-A8 message it stops timer TA8-setup.

p. PPP connection establishment procedure and optional Mobile IP Registration on the PPP connection are performed between the AT and the PDSN. Refer to RFC 1661.

q. At this point the connection is established and packet data can flow between the AT and the PDSN. 
International Journal of Distributed and Parallel Systems (IJDPS) Vol.3, No.4, July 2012

\subsection{AT originated 1xEVDO call setup -Unsuccessful Authentication}

This section describes the call flow for a successfully authenticated AT call origination in the AN shown in Figure 3.The Detail of each step is given below [2].

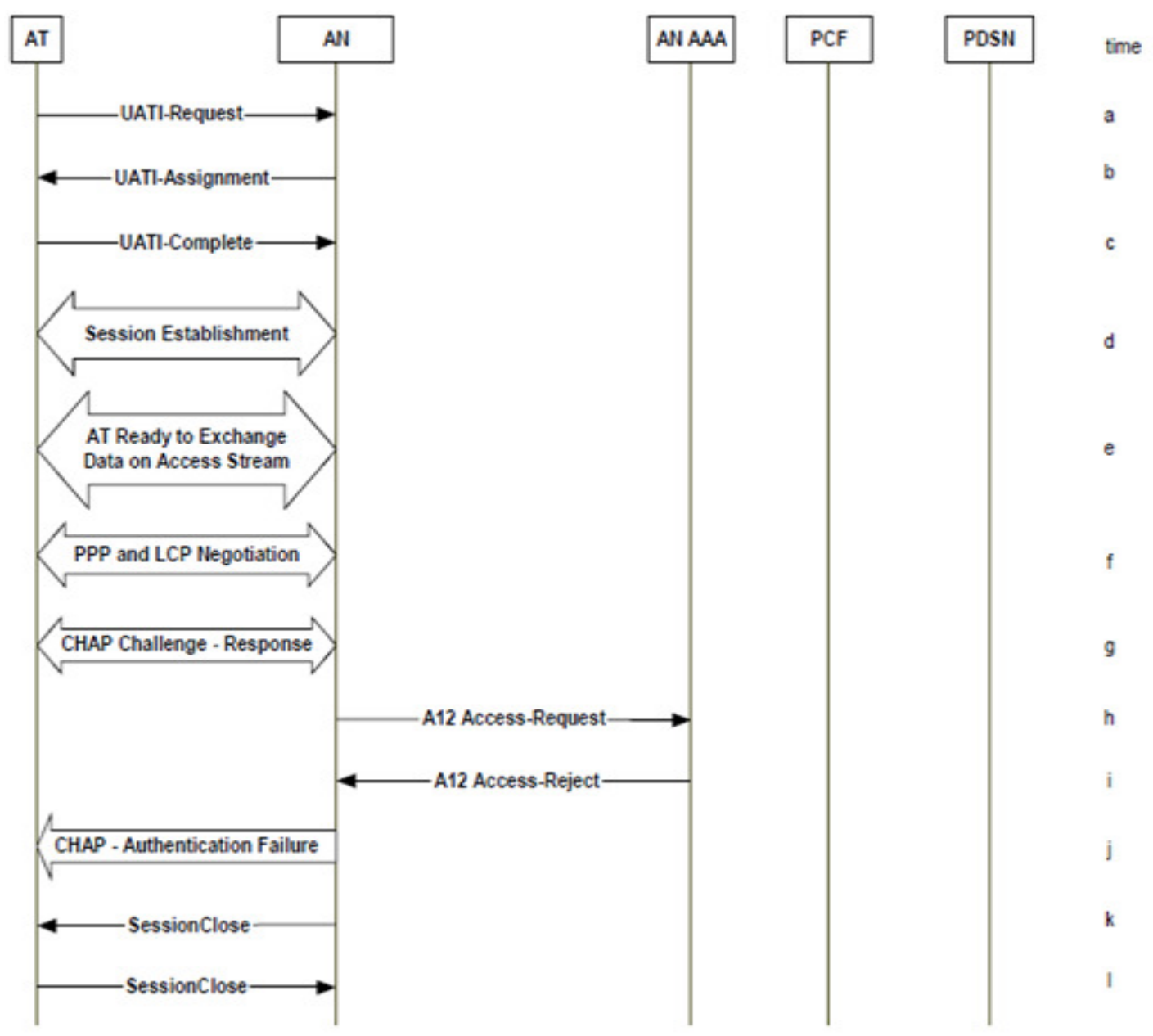

Figure 3. 1xEVDO Call Setup-Unsuccessful Authentication

a. The AT sends a UATI-Request message to request that a UATI be assigned to it by the AN.

b. The AN sends a UATI-Assignment message to assign a UATI to the AT.

c. The AT sends a UATI-Complete message to notify the AN that it has received the UATI-Assignment message.

d. If no session exists between the AT and AN, a session is established where protocols and protocol configurations are negotiated, stored and used for communications between the AT and the AN. Refer to TIA/EIA/IS-856, Section 5, Session Layer.

e. The AT indicates that it is ready to exchange data on the access stream (e.g., the flow control protocol for the default packet application bound to the AN is in the open state).

f. The AT and the AN initiate PPP and LCP negotiations for access authentication. Refer to RFC 1661. 
International Journal of Distributed and Parallel Systems (IJDPS) Vol.3, No.4, July 2012

g. The AN generates a random challenge and sends it to the AT in a CHAP Challenge packet in accordance with RFC 1994.

h. When the AN receives the CHAP response packet from the AT, it sends a RADIUS Access-Request message packet on the A12 interface to the AN AAA (which acts as a RADIUS server) in accordance with RFC-2865.

i. The AN-AAA looks up a password based on the User-1 name attribute in the A12 Access-Request and if the authentication fails (as specified in RFC-1994 and RFC2865), the AN AAA sends an Access- Reject packet on the A12 interface. Note: For ANs that perform access authentication, the network requires that no use of a dedicated resource, such as access to a PDSN, be allowed if authentication fails.

j. The AN returns an indication of CHAP authentication failure, to the AT. Refer to RFC 1994.

k. The AN sends a Session Close message to the AT, to close the session.

1. The AT responds with a Session Close message

\section{EVDO Key Performance Parameters}

In this section EVDO Key Performance Parameters are define and their formulas are given. Based on these parameters EVDO service measurements are carried out. It allows network engineer have to optimize system performance and enhance the quality of service. These KPIs are classified into following performance areas i.e. Session management, Call setup, Handoff and data throughput/capacity related parameters [4]. Each of these areas has number of parameters to measure its performance. The most important KPIs that are used by most of network operators are:

Session Setup Success Rate (SSSR): It is defined as:

SSSR $=($ Number of Successful Session setups $/$ Total Number of Session Setup Requests $) * 100$

Connection Setup Success Rate (CSSR): It is related to call/connection setup related performance parameters. It is define as:

CSSR $=($ Number of Successful connections $/$ Total number of Connection Requests $) * 100$

Blocked Call Rate: It is related to call /connection setup performance parameters. Call setup is either AT/AN initiated. It is generally defined as:

Block Call Rate $=($ Number of block calls /Total number of calls $) * 100$

Dropped Call Rate: It is also related to call / connection setup performance parameters. It is generally defined as:

Dropped Call Rate $=($ Number of drop calls $/$ Total number of Calls $) * 100$

Handoff Success Rate: It is related to handoff related performance parameters. In EVDO it is embodied by AN soft handoff on reverse link. It is generally define as:

Handoff Success Rate = (Number of Successful soft/softer handoffs / Total Handoff attempts $)$ $* 100$ 
International Journal of Distributed and Parallel Systems (IJDPS) Vol.3, No.4, July 2012

In this section vendor define KPIs are presented and their comparison is carried out. We have worked on ZTE and Huawei network equipment and KPIs define by each.

\subsection{Specification of Network Equipment Used}

In PTCL core network equipment is provided by Huawei and uses ZTE equipment at access layer. Here is the specification of the equipment used during this research work.

Huawei EMS Name: Huawei iManager M2000 Version: V200R008C03

ZTE AN: BSC6680-OMCV300R and CBTS3606 V400R007

Huawei PDSN: PDSN9660V900R007ENGC05SPC300

ZTE EMS: NetNeumen M32 (ZX10 BSSD) Version: 8.0 (3.08.30.02).

\subsection{Huawei EVDO KPIs}

Huawei uses its own terminology and formula for all EVDO KPIs defined in section 3.Each KPI has number of components that are responsible for success/failure of the parameter. The details EVDO KPIs defined by Huawei are [8]:

HRPD Session Setup Success Ratio $=($ HRPD Session setup Requests [Times] $-($ HRPD Session attempt Failures (No HardwareID Request Sent)[Times] + HRPD Session attempt Failures (No UATIAssignment Sent)[Times] + HRPD Session attempt Failures (No UATI complete message received[Times] + HRPD Session attempt Failures(No HardwareID Response Received)[Times]) / HRPD Session setup Requests [Times] *100)

Connection Success Ratio $=($ Connection Requests [Times] $-($ AT/AN-Initiated Connection Failures (Call resources allocation failure)[Times] + AT/AN-Initiated Connection Failures (No $R$-TCH Detected)[Times] + AT/AN-Initiated Connection Failures (MEID Connection Reject)[Times] + AT/AN-Initiated Connection Failures (No Traffic Channel Complete received)[Times] + AT/AN-Initiated Connection Failures (Other causes)[Times]) / Connection Requests [Times]) * 100

Block Call Rate $=((A T / A N-$ Initiated Connection Failures $($ Call resources allocation failure $)$ [Times] + AT/AN-initiated Connection Failures (No R-TCH Detected) [Times] + AT/ANInitiated Connection Failures (No Traffic Channel Complete received) [Times] + AT/ANInitiated Connection Failures (Other causes) [Times]) / AT/AN-Initiated Connection Requests [Times] * 100)

Connection Drop Ratio $=($ Connection Release $(R F$ lost $)+$ Connection Release $($ Other causes $))$ / Successful Connection Times $x 100$

Soft Handoff Success Rate = Intra-BS Soft HO Success Ratio [\%] = (Intra-BS Soft HO requests EV-DO [Times] - (Intra-BS Soft HO Failures (Radio resources unavailable) EV-DO [Times] + Intra-BS Soft HO Failures (Other causes) [Times])/ Intra-BS Soft HO requests EVDO [Times]) $\times 100$

\subsection{ZTE EVDO KPIs}

ZTE also define EVDO KPIs defined in section 3 using its own terminology and formula. Each KPI has number of constituent components that are responsible for success/failure of parameter. The details EVDO KPIs defined by ZTE are [7]: 
International Journal of Distributed and Parallel Systems (IJDPS) Vol.3, No.4, July 2012

Global Session Setup Success Rate = DO: Global Session Setup. Number of Successful Session Setups /(DO: Global Session Setup. Number of Successful Session Setups +DO: Global Session Setup. Number of Failed Session Setups + DO: Global Session Setup. Number of Failed Session Setups for Blocking) * 100

Cell Net Successful Connection Rate $=(D O:$ Cell Net Call. Number of AT Success Connection + DO: Cell Net Call. Number of AN Success Connection) / (DO: Cell Call. Number of Common Originating Calls + DO: Cell Call. Number of Common Terminating Call Responses + DO: Cell Call. Number of Fast Terminating Call Responses) * 100

Global Call RF Failure Rate $=((D O:$ Global Call. Number of Failed Common Originating Calls For Access Failure + DO: Global Call. Number of Failed Common Terminating Calls For RF Access Failure + DO: Global Call. Number of Failed Fast Terminating Calls For RF Access Failure) /(DO: Global Call. Number of Common Originating Calls + DO: Global Call. Number of Common Terminating Calls Responses + DO: Global Call. Number of Fast Terminating Responses)) * 100

Global Net Drop Rate $=(D O:$ Global Release. Number of Call System Drops + DO: Global Release. Number of PDSN Releases) / (DO: Global Release. Number of Successful Call Releases + DO: Global Release. Number of Call System Drops + DO: Global Release. Number of PDSN Releases) * 100

Global Soft Handoff Success Rate = ((DO: Global Handoff. Soft Handoff Success Num in Source AN + DO: Global Handoff. Soft Handoff Success Num in Target AN + DO: Global Handoff. Softer Handoff Success Num in Source AN + DO: Global Handoff. Softer Handoff Success Num in Target AN + DO: Global Handoff. Handoff-In Handoff Success Num between AN in Target AN + DO: Global Handoff. Handoff-Out Handoff Success Num between AN in Source AN) / DO: Number of Global Soft Handoffs)* 100

\section{Comparison of HuAwei \& ZTE Evdo KPI}

An AT must establish a 1xEVDO session with a AN before it is allowed to access the 1xEVDO network. During 1xEVDo session setup process a Universal Access terminal Identifier i.e. UATI is assigned and configuration is negotiated. A PPP session setup will also be attempted. The PPP session setup may or may not succeed but it will not affect the 1xEVDO session setup.

In Figure 4 different messages that flow during 1xEVDO Session Setup is given. The first message that will flow during EVDO session setup is Universal Access Terminal Identifier (UATI) Request. The AT generates 32-bit random number also known as session seed and includes it in the UATIRequest message and sent to the AN. This session seed is identified as the Random Access Terminal Identifier (RATI) in the UATIRequest message as depicted in Figure 4 as blue mark A. Now AN upon receiving UATIRequest message will process the message and if it succeed in processing the UATIRequest message then it will send HarwareIDRequest message back to AT as shown in Figure 4 with blue message description. If it could not process UATIRequest message due to some software problem as shown in Figure 4 with red mark B and its description. No HardwareIDRequest message will be sent and it results in Session Setup Failure and session will released and session close message flows as depicted in Figure 4 with red mark $\mathrm{C}$ and description.

Referring to normal case when AN succeed in processing the UATIRequest message and sent HardwareIDRequest message to AT but after waiting for predefine time it could not receive HarwareIDResponse message from AT. This results in timeout and session is released as shown in Figure 4 as red mark D and description. Therfore UATIAssignment message will be not sent 
International Journal of Distributed and Parallel Systems (IJDPS) Vol.3, No.4, July 2012

back to AT and session is closed as shown in Figure 4 as red mark F. If all the messages successfully flow across AT and AN then finally AT will send UATIComplete message to AN as shown in Figure 4. As blue mark $G$ which results in successful 1xEVDO Session Setup. If AN does not receive UATIComplete message at predefined time it results in timeout and session is released and results in Session Setup failure as shown in Figure 4 as red mark H.

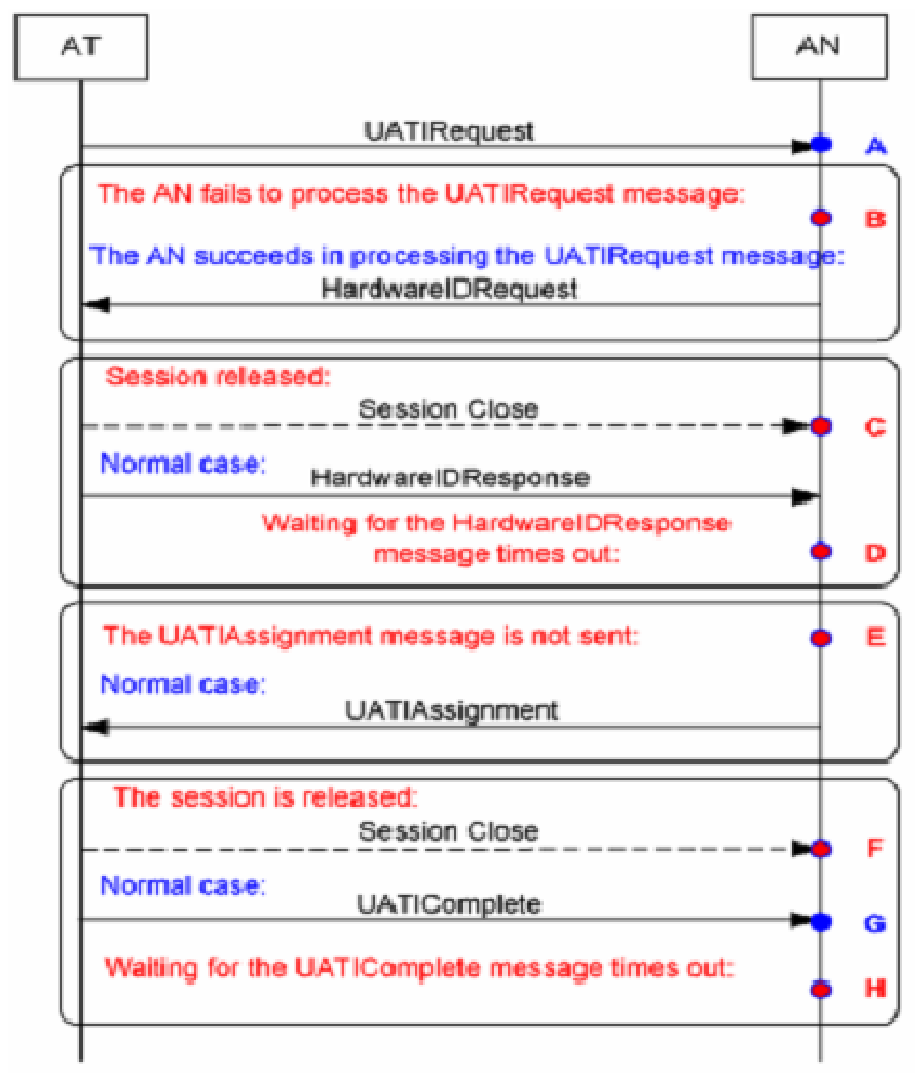

Figure 4. SSSR Message Flow

Now after discussing Session Setup messages flow we will now examine how Huawei and ZTE SSSR messages flow and what are their differences. Huawei and ZTE has almost same SSSR messages as shown in Figure 4 with differences in terminology and KPI components.

If we take Huawei and ZTE SSSR formula as given in subsection 3.2 and 3.3 and their corresponding message flow diagram in [8] and [9] we find following findings:

1. Session success $=$ UATI complete message received

2. Number of successful session setup $=$ No of UATI complete message received

3. In (Huawei) HRPD Session Setup Request (Times) = Session Setup Requests = UATIRequest In ZTE Session Setup Attempt Num = SessionSetupSNum + SessionSetupFNum + SessionSetupBlockNum.

Where:

SessionSetupSNum $=$ Session Setup Success Number 
International Journal of Distributed and Parallel Systems (IJDPS) Vol.3, No.4, July 2012

SessionSetupFNum $=$ Session Setup Failure Number

SessionSetupBlockNum $=$ Session Setup Block Number

4. We can deduct Session Setup Success Number from above formula i.e.

SessionSetupAttemptNum - SessionSetupFNum - SessionSetupBlockNum

5. Now ZTE Global Session Setup Success Rate =

((SessionSetupAttemptNum - SessionSetupFNum - SessionSetupBlockNum) / Session Setup Attempt Num) * 100)

6. Now if we compare this ZTE formula with Huawei we will find out that only SessionSetupBlockNum is additional in ZTE.

7. On the other hand if we compare both ZTE and Huawei SSSR formulae to Standard thru their respective flow diagrams we will find out that in standard SSSR is define differently than Huawei and ZTE. It calculates SSSR by subtracting failures from UATI Complete message Received. Where Number of UATI Complete Message Received = Number of Successful Session Setups (i.e. Finding No 2).

ZTE define Session Setup Block Number as

"When AN receives the UATIRequest, it fails to add new RATI and AT Index entry into the RATI and AT Index mapping table and could not sent UATI Assignment message; or when AN receives the HardwareIDResponse, it fails to add new HardwareID and AT Index entry into HardwareID and AT Index mapping table, the number of session setup block is reported. “

Unlike Huawei, ZTE separated Session Setup Failure related to Access Network to SessionSetupBlockNum i.e. AT sends all initial messages for session setup but AN could not respond to these initial session setup messages e.g. AT send UATI Request to AN but AN fail to respond to AT by not sending UATI assignment message back to AT - (According to Flow Diagram) SessionSetupBlockNum reported. Similarly if it sent UATI Assignment message to AT and then AT send UATI complete message back to AN but now AN fails to add Hardware ID into table and SessionSetupBlockNum is reported. However Huawei define all failures as Session Setup Failures whether it is related to AT or AN.

The complete set of findings and differences is shown in tabular form in Table 1. 
Table 1. Comparison of Huawei \& ZTE EVDO KPIs

\begin{tabular}{|c|c|c|c|c|}
\hline Vendor & $\begin{array}{c}\text { Vendor SSSR } \\
\text { formula Component }\end{array}$ & Reasons & Entity & Findings \\
\hline \multirow[t]{4}{*}{ Huawei } & 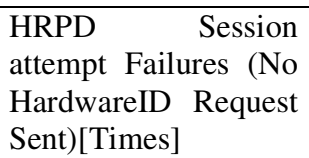 & $\begin{array}{l}\text { When AN fails to } \\
\text { process the } \\
\text { UATIRequest } \\
\text { message }\end{array}$ & AN & $\begin{array}{l}\text { Huawei term SSSR as HRPD } \\
\text { Session Setup.AT UATI request } \\
\text { message could not be processed by } \\
\text { AN due to software problem in AN }\end{array}$ \\
\hline & 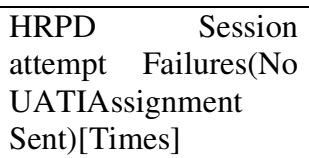 & $\begin{array}{l}\text { AN unable to send } \\
\text { the } \\
\text { UATIAssignment } \\
\text { message }\end{array}$ & AN & $\begin{array}{l}\text { AN could not send UATI } \\
\text { assignment message }\end{array}$ \\
\hline & $\begin{array}{l}\text { HRPD Session } \\
\text { attempt Failures(No } \\
\text { HardwareID } \\
\text { Response } \\
\text { Received)[Times]) }\end{array}$ & $\begin{array}{l}\text { AN is waiting for } \\
\text { the Hardware } \\
\text { IDResponse } \\
\text { message from AT } \\
\text { but timeout }\end{array}$ & AT & \\
\hline & $\begin{array}{lr}\text { HRPD } & \text { Session } \\
\text { attempt } & \text { Failures(No } \\
\text { UATI } & \text { Complete } \\
\text { Received)[Times] }\end{array}$ & $\begin{array}{l}\text { AN is waiting for } \\
\text { UATI Complete } \\
\text { message from AT } \\
\text { but timeout }\end{array}$ & AT & \\
\hline \multirow[t]{4}{*}{ ZTE } & $\begin{array}{l}\text { SessionSetupBlockN } \\
\text { um(No UATI } \\
\text { Assignment Sent }\end{array}$ & $\begin{array}{l}\text { AN unable to send } \\
\text { the } \\
\text { UATIAssignment } \\
\text { message }\end{array}$ & AN & $\begin{array}{lrr}\text { Unlike } & \text { Huawei, ZTE separated } \\
\text { Session Setup Failure due to } \\
\text { Access } & \text { Network } & \\
\text { SessionSetupBlockNum } & & \text { to }\end{array}$ \\
\hline & $\begin{array}{l}\text { SessionSetupBlockN } \\
\text { um(Fail to add } \\
\text { Hardware ID) }\end{array}$ & $\begin{array}{l}\text { AN fails to add } \\
\text { new HardwareID } \\
\text { and ATIndex } \\
\text { entry } \\
\text { HardwareID and } \\
\text { ATIndex mapping } \\
\text { table ID into }\end{array}$ & AN & $\begin{array}{l}\text { Unlike Huawei, ZTE separated } \\
\text { Session Setup Failure due to } \\
\text { Access Network to SessionSetup } \\
\text { BlockNum }\end{array}$ \\
\hline & $\begin{array}{l}\text { SessionSetupFNum( } \\
\text { No UATI Complete } \\
\text { Received) }\end{array}$ & $\begin{array}{l}\text { AN is waiting for } \\
\text { UATI Complete } \\
\text { message from AT } \\
\text { but timeout }\end{array}$ & AT & $\begin{array}{l}\text { Same as Huawei HRPD Session } \\
\text { attempt } \quad \text { Failures(No UATI } \\
\text { Complete Received)[Times] }\end{array}$ \\
\hline & $\begin{array}{l}\text { SessionSetupFNum( } \\
\text { No HardwareID } \\
\text { Response Received) }\end{array}$ & $\begin{array}{l}\text { AN is waiting for } \\
\text { the Hardware } \\
\text { IDResponse } \\
\text { message from AT } \\
\text { but timeout }\end{array}$ & AT & $\begin{array}{l}\text { Same as Huawei HRPD Session } \\
\text { attempt Failures(No HardwareID } \\
\text { Response Received)[Times]) }\end{array}$ \\
\hline
\end{tabular}




\section{UNifiCATION OF MULTIVENDOR DEFINED KPI}

In this section we will unify SSSR defined by different vendors i.e. Huawei and ZTE. For this purpose we will include Session Negotiation Failure into both Huawei and ZTE SSSR KPI formulae because it is operator responsibility to ensure proper and right AT availability in CDMA network. Since a bad terminal can destroy whole cell communication e.g. because of excess transmit power which is considered as noise for other terminals. During Session Configuration phase AT and AN negotiate a set of protocol subtypes, application subtypes and the relevant parameters for each personality. So now SSSR for both Huawei becomes:

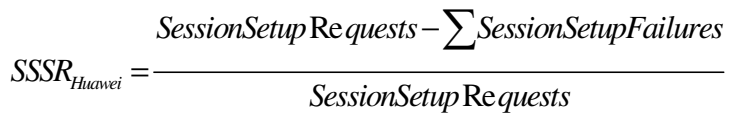

Where:

SessionSetupRequests $=$ HRPD Session Setup Requests

$\sum$ SessionSetupFailures $=$ HRPD Session attempt Failures $($ No HardwareID Request Sent $)+$ HRPD Session attempt Failures (No UATIAssignment Sent) + HRPD Session attempt Failures (No UATI complete message received + HRPD Session attempt Failures(No HardwareID Response Received + HRPDSessionConfNegoFailure)

And that of ZTE become:

$S S S R_{Z T E}=\gamma\left(\frac{\text { SessionSetup } \text { Re quests }-\sum \text { SessionSetupFailures }}{\text { SessionSetup } \text { Re quests }}\right)$

Where:

$\sum$ SessionSetupFailures $=$ SessionSetupFNum + SessionSetupBNum + SessionNegoFNum

SessionSetupRequests $=$ SessionSetupAttemptNum

And

$\gamma=$ CorrectionFactor

In order to get a meaningful and more comprehensive view of system QoS we define a new KPI HSN Ratio (Harmonic mean of session Setup success and session Negotiation success Ratios). The harmonic mean is often used as an aggregated performance score of two or more quality indicators [11]. We will introduce a constant $\gamma$ in Huawei HSN formula which is used to minimize both ZTE and Huawei differences so that we can unify them. The value of this correction factor $\gamma$ will be calculated by Minimum Mean Square Error (MMSE) Method.

$F(\gamma)=\sum_{i}\left(\left(\text { HuaweiHSN }_{i}+\gamma\right)-Z T E H S N_{i}\right)^{2}$ 
Then, differentiating $F(\gamma)$ relative to $\gamma$ and setting it to zero

$\frac{\partial F(\gamma)}{\partial \gamma}=0$

\section{NUMERICAL RESULTS}

We have taken performance statistic report for both KPI's i.e. SSSR and SNR from Huawei M2000 iManager and ZTE CNO2 for 15 days. These statistic reports contain values of each constituent counter of SSSR and SNR calculated for each hour for 15 days. We have first calculated average SSSR and SNR by taking average of SSSR and SNR values of each hour for 15 days. This results in 24 average hour data each for Huawei SSSR \& SNR. Similar procedure is repeated for ZTE and calculated 24 average hour data each for ZTE SSSR \& SNR.

Next we calculated harmonic mean of this average hour SSSR and SNR for both ZTE and Huawei. This results in a new KPI HSN (i.e. harmonic mean of SSSR and SNR) each for Huawei and ZTE. These Huawei HSN and ZTE HSN values are put into the formula:

$$
F(\gamma)=\sum_{i}\left(\left(\text { HuaweiHSN }_{i}+\gamma\right)-\text {ZTEHSN }_{i}\right)^{2}
$$

This yield up with $2^{\text {nd }}$ order equation in terms of $\gamma$. This is finally differentiated with respect to $\gamma$ to find value for it:

$\gamma=-0.097$

To prove our results we have drawn graphs for Huawei_HSN and ZTE_HSN as shown in Fig.5.

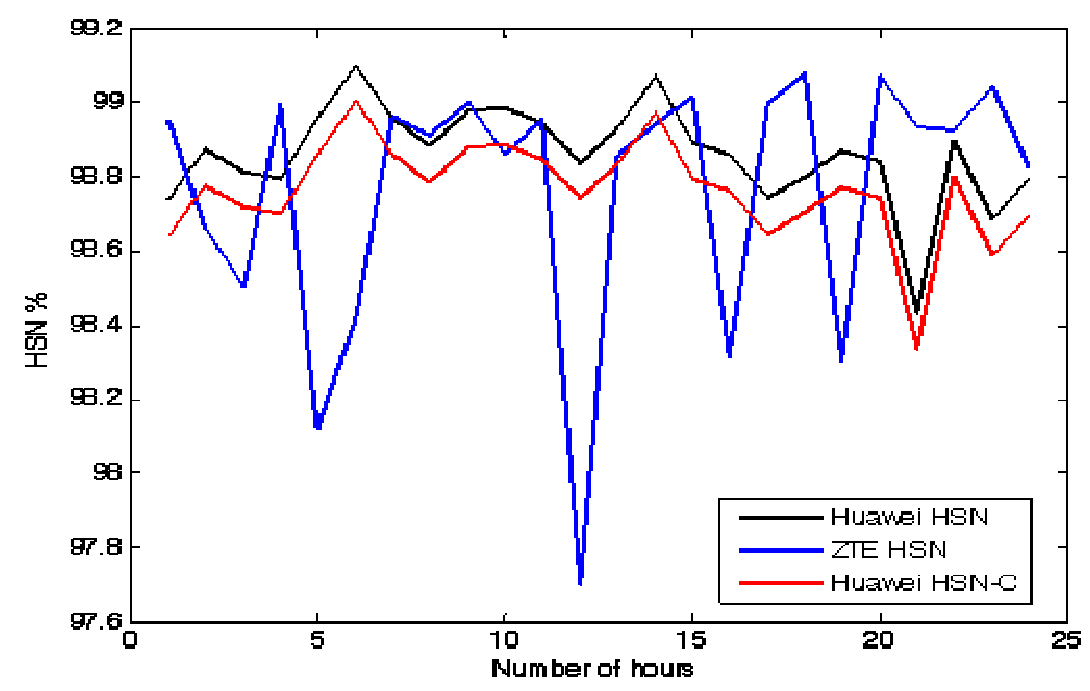

Figure 5. Huawei \& ZTE HSN 
International Journal of Distributed and Parallel Systems (IJDPS) Vol.3, No.4, July 2012

We have then drawn the difference graphs before and after correction as shown in Figure 6 and 7. In Figure 6 the Mean value of difference between Huawei_HSN and ZTE_HSN before correction $=-0.09701$. In Figure 7 difference between Huawei_HSN and ZTE_HSN is redrawn after correction applied which minimizes the mean to $7.9 \mathrm{e}-6$ which is nearly equal to 0 .

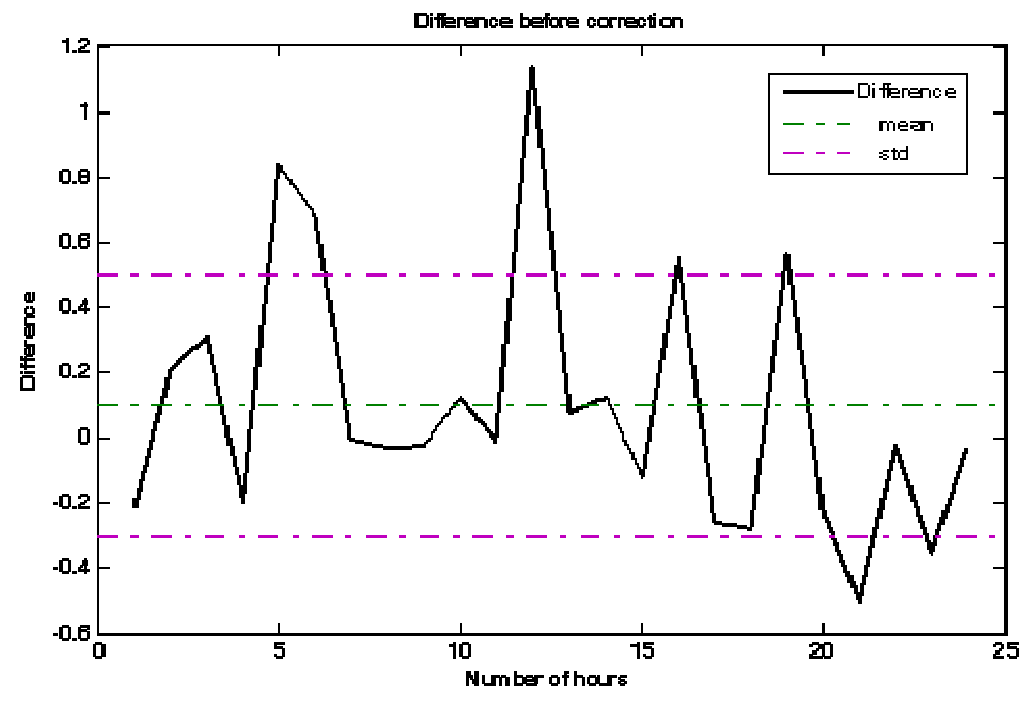

Figure 6. Difference Before Correction

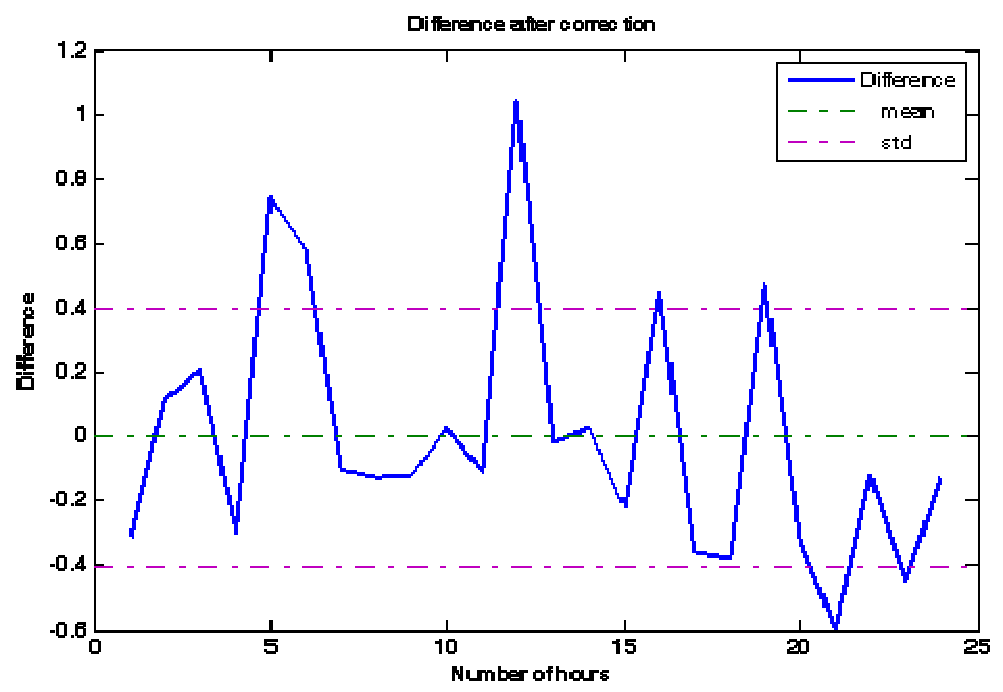

Figure 7. Difference After Correction

In Figure 8 and 9 we have drawn absolute difference graphs of Huawei and ZTE HSN. The standard deviation (STD) before correction is equal to 0.2911 as shown in Figure 8 and STD after correction is equal to 0.2529 . Hence we have minimizes the difference between Huawei and ZTE SSSR 


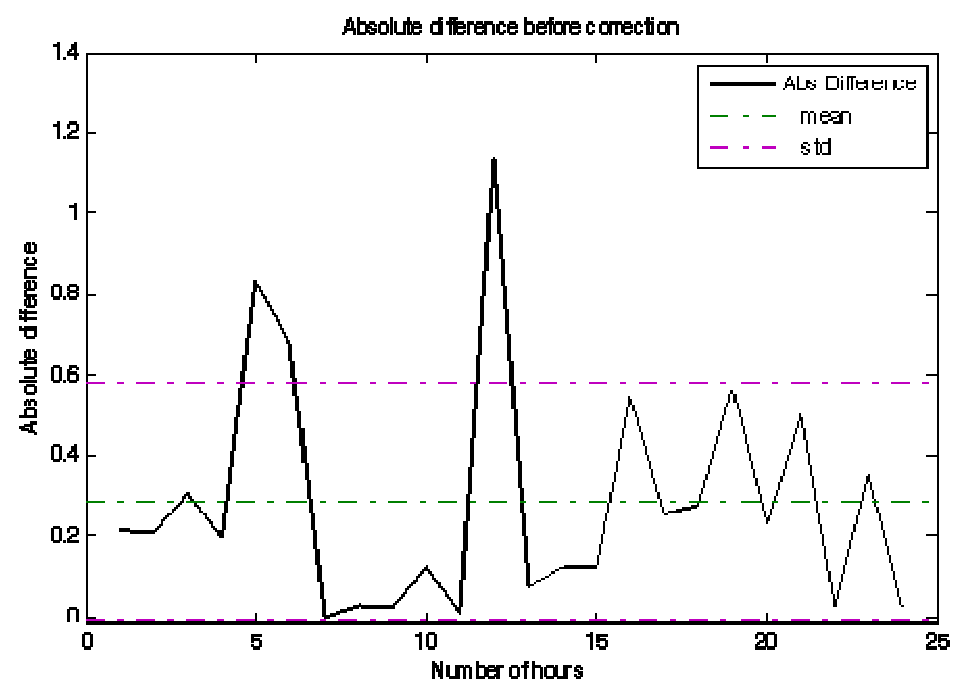

Figure 8. Absoloute Difference Before Correction

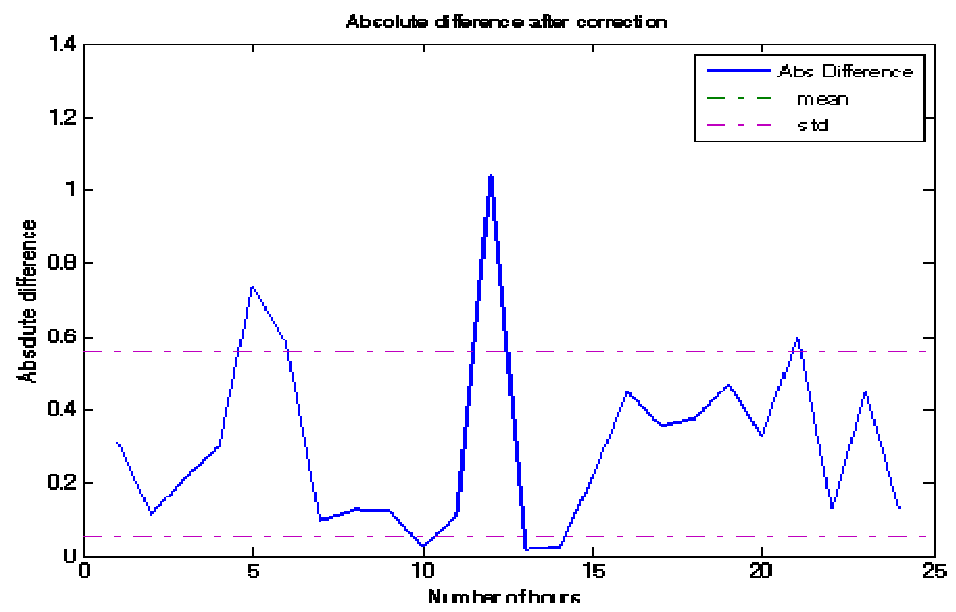

Figure 9. Absoloute Difference After Correction

\section{CONClusions}

The problem of different EVDO KPIs defined by different vendors in multivendor environment could be unified by minimizing their differences and introducing some new KPIs. We have done this for most important EVDO KPI which is session setup success rate also known as SSSR. In future same method and techniques could be applied to unify all EVDO KPIs. This will lead to one set of EVDO KPIs defined for all different vendors and hence will be very helpful for network optimization engineers to analyze system performance and quality of service. 
International Journal of Distributed and Parallel Systems (IJDPS) Vol.3, No.4, July 2012

\section{ACKNOWLEDGEMENTS}

Authors would like to thank to Network Operation Center (NOC) team at Pakistan Telecommunication Company, for their support and access to EVDO data.

\section{REFERENCES}

[1] 3rd Generation Partnership Project 2, "CDMA2000 High Rate Packet Data Air Interface Specification,” TIA/EIA/IS-856, Version 2.0, C.S0024, Oct. 27, 2000, <http://www.3gpp2.org/>

[2] 3GPP2 A..S0007 Ballot version "1xEVDO Inter-Operability Specification(IOS) for CDMA2000 Access Network Interfaces” June 14,2001.

[3] Naga Bhushan, Chris Lott, Peter Black, Rashid Attar, Yu-Cheun Jou, Mingxi Fan, Donna Ghosh, and Jean Au, QUALCOMM, Inc. "CDMA2000 1xEV-DO Revision A: A Physical Layer and MAC Layer Overview", IEEE Communications Magazine 2006

[4] Frances Jiang “Lucent 1xEVDO Service Measurements for Wireless Teams" Dec 2008.

[5] 3GPP2 A.S0009-C_v2.0 "Interoperability Specification for High Rate Packet Data (HRPD) Radio Access Network Interfaces with Session Control in Packet Control Function" January 2009

[6] QUALCOMM “1xEV-DO Revision A Session Negotiation Example” March 102008

[7] ZTE ZXC10 BSSBCDMA Base Station System "EVDO Performance Management Index Description" Sept 2008

[8] HUAWEI “CDMA2000 1xEVDO Rev A Performance Analysis” 2009

[9] Dr Enest Simo "1xEVDO: Rev 0 \& Rev A"

[10] Abramowitz, M. and Stegun, I. A. (Eds.). Handbook of Mathematical Functions with Formulas, Graphs, and Mathematical Tables, 9th 